\title{
The use of LCMV-specific T cell hybridomas for the quantitative analysis of MHC class I restricted antigen presentation
}

\author{
Katrin Schwarz ${ }^{\mathrm{a}}$, Maries van den Broek ${ }^{\mathrm{b}}$, Rita de Giuli ${ }^{\mathrm{a}}$, Wolfhart W. Seelentag ${ }^{\mathrm{c}}$, \\ Nilabh Shastri ${ }^{\mathrm{d}}$, Marcus Groettrup ${ }^{\mathrm{a}, *}$ \\ ${ }^{a}$ Research Department, Cantonal Hospital St. Gall, Building 09, CH-9007 St. Gallen, Switzerland \\ ${ }^{\mathrm{b}}$ Institute of Experimental Immunology, Department of Pathology, University Hospital Zürich, CH-8091 Zürich, Switzerland

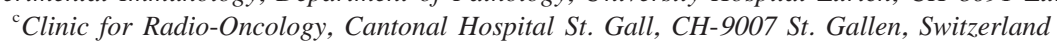 \\ ${ }^{\mathrm{d}}$ Division of Immunology, Department of Molecular and Cell Biology, University of California, Berkeley, CA 94720-3200, USA
}

Keywords: LCMV; Cytotoxic T lymphocyte; Hybridoma; Proteasome; Antigen presentation

Somatic T cell hybridomas can be used to monitor the processing and presentation of $\mathrm{T}$ cell antigens in the MHC class I pathway. Here we report the generation of hybridomas by fusion of three cytotoxic $\mathrm{T}$ lymphocyte (CTL) lines specific for different epitopes of the lymphocytic choriomeningitis virus (LCMV) glycoprotein and nucleoprotein. The cell line BWZ.36.1/CD8 $\alpha$ used as a fusion partner is a $\mathrm{T}$ cell receptor-deficient lymphoma line (BW5147 $\alpha^{-} \beta^{-}$) expressing CD8 $\alpha$ and a lacZ reporter construct under the control of the IL-2 promotor/ enhancer. This system permits monitoring of $\mathrm{T}$ cell

Abbreviations: APC, antigen presenting cell; CTL, cytotoxic T lymphocyte; GP, glycoprotein; LCMV, lymphocytic choriomeningitis virus; MHC, major histocompatibility complex; MOI, multiplicity of infection; NP, nucleoprotein; OVA, ovalbumin; TAP, transporter associated with antigen processing; TCR, T cell receptor

*Corresponding author. Tel.: +41-71-494-1069; fax: +41-71494-6321.

E-mail address: lfal@ms1.kssg.ch (M. Groettrup) hybrid stimulation in chromogenic lacZ assays. A comparison of the sensitivity of the LCMV-specific hybridomas in the lacZ assay with the performance of the parental CTL lines in chromium release assays revealed a $10^{2}$ to $10^{4}$-fold lower sensitivity of the hybridoma-based lacZ assay. Accordingly, the recognition of the LCMV-glycoprotein epitope GP33 by a GP33-specific hybridoma was reduced by the proteasome specific inhibitor lactacystin to a greater extent and at a lower concentration than CTL recognition in a chromium release assay. Hence, the hybridomabased lacZ assay is complementary to the chromium release assay in that it covers a lower range of sensitivity which allows the detection of subtle differences in the efficiency of antigen processing due to the induction of proteasome subunits or other components of the MHC class I pathway.

The lymphocytic choriomeningitis virus (LCMV)mouse system is one of the best characterized systems with which to study the cytotoxic immune response in vivo and in vitro. Three immuno- 
dominant $\mathrm{T}$ cell epitopes of the virus are presented by the $H-2 D^{b}$ class I molecule: the glycoprotein (GP) derived peptide epitopes GP33 (KAVYNFATC) and GP276 (SGVENPGGYCL) as well as the nucleoprotein (NP)-derived epitope NP396 (FQPQNGQFI) (Gallimore et al., 1998a). For the $\mathrm{H}-2 \mathrm{~L}^{\mathrm{d}}$ restriction element the immunodominant epitope NP118 (RPQASGVYM) has been delineated. The intracellular processing of these four epitopes appears to be dependent on the proteasome since antigen presentation is prevented by the specific proteasome inhibitor lactacystin (Gallimore et al., 1998b). To date, antigen presentation in this system has been analyzed by conventional cytolytic chromium release assays which are extremely sensitive with respect to peptide concentration $\left(10^{-8}\right.$ $10^{-13} \mathrm{M}$ ) and require the generation of cytotoxic $\mathrm{T}$ lymphocyte (CTL) lines from LCMV infected mice by periodic restimulation. The laborious maintenance of oligoclonal CTL lines, their variability in culture, and the enormous sensitivity of chromium release assays are not ideal prerequisites for monitoring the efficiency of antigen processing by the proteasome because differences in epitope generation (due for instance to interferon- $\gamma$ inducible exchanges of proteasome subunits and regulators) may be in the micro- to nano- rather than in the nano- to picomolar range of detection.

We therefore generated somatic $\mathrm{T}$ cell hybridomas specific for the $\mathrm{H}-2 \mathrm{D}^{\mathrm{b}}$ restricted epitopes GP33 and GP276 as well as the $\mathrm{H}-2 \mathrm{~L}^{\mathrm{d}}$ restricted epitope NP118. Oligoclonal CTL lines were obtained from LCMV infected C57BL/ 6 and BALB/c mice by weekly restimulation as described (Gallimore et al., 1998b) and after the third restimulation the cells were fused with BWZ.36.1/CD8 $\alpha$ cells as detailed elsewhere (Sanderson and Shastri, 1994). This fusion partner expresses transfected cDNAs for CD8 $\alpha$ and the bacterial $\beta$-galactosidase (lacZ) reporter gene under the transcriptional control of the IL-2 promotor and the nuclear factor of activated T cells (NFAT)element of the human IL-2 enhancer. Antigenic stimulation of the generated LCMV-specific $\mathrm{T}$ cell hybridomas can thus be easily monitored in chromogenic lacZ assays as described (Sanderson and Shastri, 1994).

Three hybridoma clones derived from CTLs specific for GP33/H-2D ${ }^{\mathrm{b}}$, GP276/H-2D ${ }^{\mathrm{b}}$, or NP118/H-
2L ${ }^{\mathrm{d}}$ which were positive for CD3 and CD8 molecules in flow cytometric analysis were selected for further characterization. The specificity of the hybridomas both for the respective restriction element and for the appropriate epitope was determined in lacZ assays using LCMV infected or peptide loaded fibroblast lines of $\mathrm{H}-2^{\mathrm{b}}$ (MC57) and $\mathrm{H}-2^{\mathrm{d}}$ (B8) haplotypes as targets. In addition a quantitative analysis comparing the detection limits between CTLs and hybridomas was performed (Fig. 1A). RMA-S cells $\left(\mathrm{H}-2^{\mathrm{b}}\right)$ loaded with GP33 peptide or T2-L ${ }^{d}$ cells loaded with NP118 peptide at concentrations between $10^{-5}$ and $10^{-12} \mathrm{M}$ were used as targets for hybridomas and CTLs of the respective specificity. The CTL-mediated lysis of target cells loaded with a peptide concentration as low as $10^{-12}$ $\mathrm{M}$ peptide was detectable with maximal lysis being reached at $10^{-8} \mathrm{M}$ peptide. In contrast, the hybridomas needed $10^{2}-10^{4}$ times higher peptide concentrations to show activation in the lac Z assay. Measuring activation of hybridomas started at $10^{-7}-$ $10^{-8} \mathrm{M}$ concentrations of peptide and increased with higher concentrations without reaching a maximum even above $10^{-5} \mathrm{M}$ peptide. All of the LCMVspecific hybridomas tested in this study were 100 to 10,000-fold less sensitive when compared to parental CTL lines, which is significantly greater than the 10-fold drop in sensitivity previously found for an ovalbumin/H-2K ${ }^{\mathrm{d}}$-specific hybridoma (Shastri and Gonzalez, 1993).

As the effector to target (E:T) ratio is used in cytolytic assays to titrate the lytic effect we similarly titrated the E:T ratio with our LCMV-specific T cell hybridomas. The result shown for the GP33-specific CTL line and corresponding hybridoma in Fig. 1B indicates that the lacZ assay likewise responds to the $\mathrm{E}: \mathrm{T}$ ratio. In contrast to the cytolytic assay which reaches a plateau at an E:T ratio of 10 the colour reaction of the lacZ assay displays a steady increase of absorbance for E:T values ranging from 2 to 30 .

The lower range of sensitivity in the hybridomabased lacZ assay would suggest that deficiencies in the proteasome-dependent generation of $\mathrm{T}$ cell epitopes should become apparent in the lacZ assay earlier than in the chromium release assay. In order to test this hypothesis we treated MC57 fibroblasts with different concentrations of the proteasome-specific inhibitor lactacystin prior to infection with a 

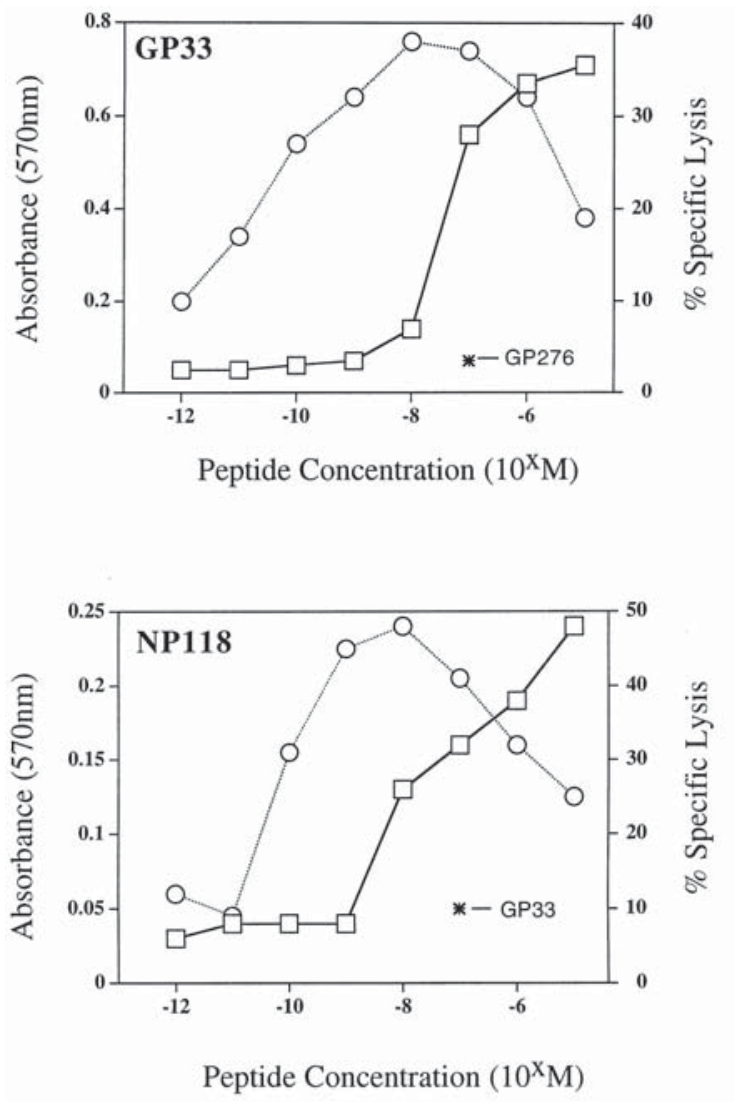

(a)
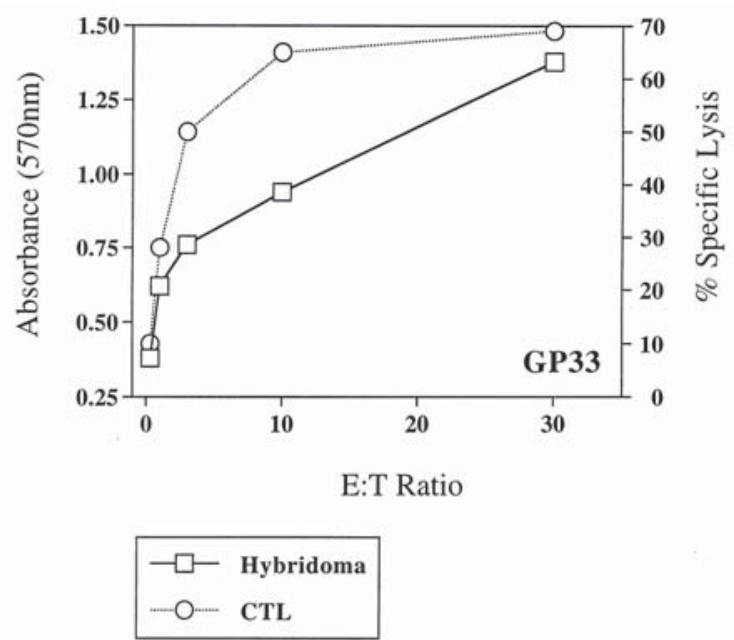

(b)

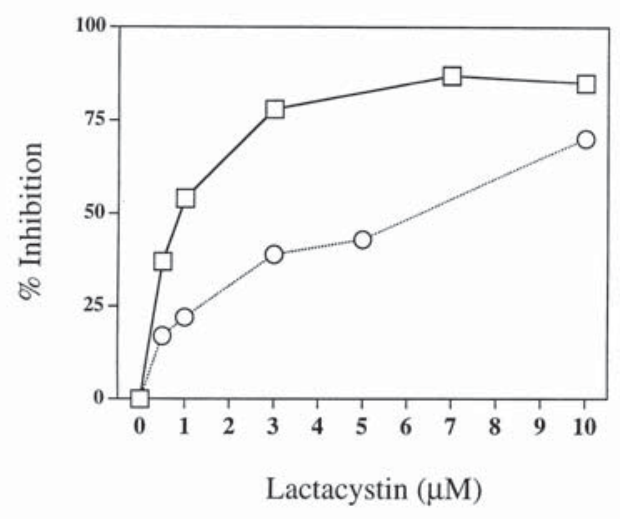

(c)

Fig. 1. Test of peptide specificity and sensitivity of hybridomas in the lacZ assay (open boxes) and comparison with parental CTL lines in chromium release assays (open circles). (A) Peptide titration for GP33 and NP118-specific hybridomas and CTLs: RMA-S (H-2 ${ }^{\mathrm{b}}$ ) or T2-L ${ }^{\mathrm{d}}$ cells were pulsed with titrated concentrations of the respective peptides and used as target cells in either a lacZ assay or in a chromium release assay. The left hand axis shows absorbance of enzymatically converted chromogen at $570 \mathrm{~nm}$ in lacZ assays, the right hand axis shows the specific chromium release in cytolytic assays. The absorbance obtained by presentation of an irrelevant peptide at a concentration of $0.1 \mu \mathrm{M}$ is indicated in the upper (GP276) and lower (GP33) panel. (B) Dependence on effector to target (E:T) ratio: MC57 fibroblasts were infected with LCMV-WE at a MOI of 0.01 for $48 \mathrm{~h}$ before they were used as targets for lysis by GP33-specific CTLs or as stimulators in a lacZ assay. (C) The effect of titrated amounts of lactacystin on GP33 antigen presentation as monitored in lacZ and chromium release assays. MC57 cells were pretreated with the indicated concentrations of lactacystin for 2 h prior to infection with recombinant vaccinia virus rVVG2 encoding the LCMV glycoprotein (Gallimore et al., 1998b) at a MOI of 5. After $1.5 \mathrm{~h}$ the cells were used as targets in a standard $5 \mathrm{~h}$ chromium release assay or a lacZ assay.

recombinant vaccinia virus expressing the LCMV glycoprotein. The presentation of the GP33 epitope was then measured both by CTLs in a chromium release assay and with our GP33 reactive hybridoma in a lacZ assay. Indeed, GP33 recognition by the hybridoma was affected to a greater extent and at lower concentrations of lactacystin than CTL-mediated lysis (Fig. 1C). At a concentration of $1 \mu \mathrm{M}$ lactacystin, hybridoma activation was consistently inhibited by $50-60 \%$ whereas the inhibition of 
specific lysis in chromium release assays ranged from 0 to $20 \%$ depending on the CTL line used. While hybridoma recognition of the GP33 epitope was virtually abolished at $3 \mu \mathrm{M}$ lactacystin the chromium release was still further impeded in a dose-dependent manner up to $10 \mu \mathrm{M}$ lactacystin. These results indicate that hybridomas are better suited to monitor minor deficiencies in antigen processing while CTLs are superior in the quantitative evaluation of more severe defects in antigen presentation.

Although the hybridoma-based lacZ assay cannot be used to study the effector functions of CTLs, this read out system offers many advantages: it is a monoclonal system with invariant specificity; the hybridomas can be thawed shortly before assay performance and grow rapidly and to large numbers without the need for feeder cells, cytokines, or restimulations. The lacZ assay is cheap, fast, and non-radioactive. Most importantly, however, the lower sensitivity of our LCMV-specific hybridomas allows us to monitor differences in antigen processing which may previously have remained unnoticed in chromium release assays and which nevertheless may be of physiological significance for the success of an antiviral cytotoxic immune response.

\section{Acknowledgements}

We thank Ferry Ossendorp and Stephen Schoenberger for providing BWZ.36.1/CD8 $\alpha$ cells and Thomas Ruppert for $\mathrm{T}_{2} \mathrm{~L}^{\mathrm{d}}$ cells. This work was supported by a grant of the Swiss National Science Foundation (31-52284.97/1), by the Roche Research Foundation, Novartis Foundation, and Rentenanstalt Jubiläumsstiftung.

\section{References}

Gallimore, A., Dumrese, T., Hengartner, H., Zinkernagel, R.M., Rammensee, H.-G., 1998a. Protective immunity does not correlate with the hierarchy of virus-specific cytotoxic $\mathrm{T}$ cell responses to naturally processed peptides. J. Exp. Med. 187, 1647-1657.

Gallimore, A., Schwarz, K., van den Broek, M., Hengartner, H., Groettrup, M., 1998b. The proteasome inhibitor lactacystin prevents the generation of an endoplasmic reticulum leaderderived T cell epitope. Mol. Immunol. 35, 581-591.

Sanderson, S., Shastri, N., 1994. LacZ inducible, antigen/MHCspecific T cell hybrids. Int. Immunol. 6, 369-376.

Shastri, N., Gonzalez, F., 1993. Endogenous generation and presentation of the ovalbumin peptide $/ \mathrm{K}^{\mathrm{b}}$ complex to $\mathrm{T}$ cells. J. Immunol. 150, 2724-2736. 